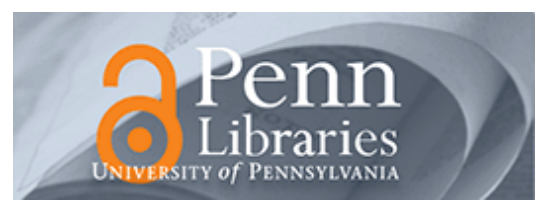

University of Pennsylvania

ScholarlyCommons

$10-2014$

\title{
PTH1-34 Alleviates Radiotherapy-Induced Local Bone Loss by Improving Osteoblast and Osteocyte Survival
}

\author{
Abhishek Chandra \\ University of Pennsylvania \\ Tiao Lin \\ University of Pennsylvania \\ Mary Beth Tribble \\ University of Pennsylvania \\ Jhi Zhu \\ University of Pennsylvania \\ Allison R. Altman \\ University of Pennsylvania
}

See next page for additional authors

Follow this and additional works at: https://repository.upenn.edu/dental_papers

Part of the Dentistry Commons

\section{Recommended Citation}

Chandra, A., Lin, T., Tribble, M. B., Zhu, J., Altman, A. R., Tseng, W., Zhang, Y., Akintoye, S. O., Cengel, K., Liu, X. S., \& Qin, L. (2014). PTH1-34 Alleviates Radiotherapy-Induced Local Bone Loss by Improving Osteoblast and Osteocyte Survival. Bone, 6733-40. http://dx.doi.org/10.1016/j.bone.2014.06.030

This paper is posted at ScholarlyCommons. https://repository.upenn.edu/dental_papers/76

For more information, please contact repository@pobox.upenn.edu. 


\title{
PTH1-34 Alleviates Radiotherapy-Induced Local Bone Loss by Improving Osteoblast and Osteocyte Survival
}

\begin{abstract}
Cancer radiotherapy is often complicated by a spectrum of changes in the neighboring bone from mild osteopenia to osteoradionecrosis. We previously reported that parathyroid hormone (PTH, 1-34), an anabolic agent for osteoporosis, reversed bone structural deterioration caused by multiple microcomputed tomography (microCT) scans in adolescent rats. To simulate clinical radiotherapy for cancer patients and to search for remedies, we focally irradiated the tibial metaphyseal region of adult rats with a newly available small animal radiation research platform (SARRP) and treated these rats with intermittent injections of PTH1-34. Using a unique 3D image registration method that we recently developed, we traced the local changes of the same trabecular bone before and after treatments, and observed that, while radiation caused a loss of small trabecular elements leading to significant decreases in bone mass and strength, PTH1-34 preserved all trabecular elements in irradiated bone with remarkable increases in bone mass and strength. Histomorphometry demonstrated that SARRP radiation severely reduced osteoblast number and activity, which were impressively reversed by PTH treatment. In contrast, suppressing bone resorption by alendronate failed to rescue radiation-induced bone loss and to block the rescue effect of PTH1-34. Furthermore, histological analyses revealed that PTH1-34 protected osteoblasts and osteocytes from radiation-induced apoptosis and attenuated radiation-induced bone marrow adiposity. Taken together, our data strongly support a robust radioprotective effect of PTH on trabecular bone integrity through preserving bone formation and shed light on further investigations of an anabolic therapy for radiation-induced bone damage.
\end{abstract}

\section{Keywords}

parathyroid hormone, radiotherapy, image registration, trabecular bone, osteoblast, apoptosis

Disciplines
Dentistry

Author(s)

Abhishek Chandra, Tiao Lin, Mary Beth Tribble, Jhi Zhu, Allison R. Altman, Weiju Tseng, Yejia Zhang, Sunday O. Akintoye, Keith Cengel, X. Sherry Liu, and Ling Qin 


\title{
PTH1-34 Alleviates Radiotherapy-induced Local Bone Loss by Improving Osteoblast and Osteocyte Survival
}

\author{
Abhishek Chandraa ${ }^{a}{ }^{,}$, Tiao Lin ${ }^{a}, b,{ }^{,}$, Mary Beth Tribblea ${ }^{a}$, Ji Zhua ${ }^{a}$, Allison R. Altman ${ }^{a}$, Weiju \\ Tseng $^{\mathrm{a}}$, Yejia Zhang ${ }^{\mathrm{c}}$, Sunday O. Akintoye ${ }^{\mathrm{d}}$, Keith Cengel $^{\mathrm{e}}$, X. Sherry Liu ${ }^{\mathrm{a}}$, and Ling Qin ${ }^{\mathrm{a}, 1}$ \\ aDepartment of Orthopaedic Surgery, Perelman School of Medicine, University of Pennsylvania, \\ Philadelphia, Pennsylvania, 19104, USA \\ bDepartment of Orthopaedic Surgery, Second Affiliated Hospital, School of Medicine, Zhejiang \\ University, Hangzhou, 310009, China \\ cPhiladelphia Veterans Affairs Medical Center and Department of Physical Medicine and \\ Rehabilitation, Perelman School of Medicine, University of Pennsylvania, Philadelphia, PA \\ dDepartment of Oral Medicine, Penn Dental School, University of Pennsylvania, Philadelphia, \\ Pennsylvania, 19104, USA \\ eDepartment of Radiation Oncology, Perelman School of Medicine, University of Pennsylvania, \\ Philadelphia, Pennsylvania, 19104, USA
}

\begin{abstract}
Cancer radiotherapy is often complicated by a spectrum of changes in the neighboring bone from mild osteopenia to osteoradionecrosis. We previously reported that parathyroid hormone (PTH, 134), an anabolic agent for osteoporosis, reversed bone structural deterioration caused by multiple microcomputed tomography (microCT) scans in adolescent rats. To simulate clinical radiotherapy for cancer patients and to search for remedies, we focally irradiated the tibial metaphyseal region of adult rats with a newly available small animal radiation research platform (SARRP) and treated these rats with intermittent injections of PTH1-34. Using a unique 3D image registration method that we recently developed, we traced the local changes of the same trabecular bone before and after treatments, and observed that, while radiation caused a loss of small trabecular elements leading to significant decreases in bone mass and strength, PTH1-34 preserved all trabecular elements in irradiated bone with remarkable increases in bone mass and strength.

Histomorphometry demonstrated that SARRP radiation severely reduced osteoblast number and activity, which were impressively reversed by PTH treatment. In contrast, suppressing bone resorption by alendronate failed to rescue radiation-induced bone loss and to block the rescue effect of PTH1-34. Furthermore, histological analyses revealed that PTH1-34 protected
\end{abstract}

(C) 2014 Elsevier Inc. All rights reserved.

${ }^{1}$ Corresponding author: Ling Qin, Department of Orthopaedic Surgery, University of Pennsylvania, 424A Stemmler Hall, $36^{\text {th }}$ Street and Hamilton Walk, Philadelphia, PA 19104, USA, Tel: 215-8986697; Fax: 215-5732133; qinling@ mail.med.upenn.edu.

These authors contribute equally to this article.

Publisher's Disclaimer: This is a PDF file of an unedited manuscript that has been accepted for publication. As a service to our customers we are providing this early version of the manuscript. The manuscript will undergo copyediting, typesetting, and review of the resulting proof before it is published in its final citable form. Please note that during the production process errors may be discovered which could affect the content, and all legal disclaimers that apply to the journal pertain. 
osteoblasts and osteocytes from radiation-induced apoptosis and attenuated radiation-induced bone marrow adiposity. Taken together, our data strongly support a robust radioprotective effect of PTH on trabecular bone integrity through preserving bone formation and shed light on further investigations of an anabolic therapy for radiation-induced bone damage.

\section{Keywords}

parathyroid hormone; radiotherapy; image registration; trabecular bone; osteoblast; apoptosis

\section{Introduction}

Radiation therapy has more than 100 years of history as a cancer treatment. Each year, about 1 million cancer patients are prescribed radiotherapy in conjunction with surgery and chemotherapy in order to eliminate tumor cells $[1,2]$. The effectiveness of radiotherapy is based on the radiation dosage delivered to a tumor and is limited by the radiation tolerance of its surrounding normal tissues. Radiation damage to the skeleton within the radiation field is a well-recognized late effect, resulting in a spectrum of bone changes from mild osteopenia to osteoradionecrosis [3-5]. To date, the mechanism of radiation-induced bone damage has not been fully elucidated. The improved survivorship rate and the increased age of cancer patients emphasize the importance of understanding this mechanism and identifying an effective treatment to prevent or reverse such skeletal damage. Currently, anti-resorptive drugs, such as bisphosphonates, are sometimes used to treat the radiationinduced osteoporosis, but the evidence of clinical efficacy for this approach is limited and inconclusive. Moreover, long term use of bisphosphonates is associated with risks, such as osteonecrosis of the jaw and atypical femur fractures.

Bone is a dynamic organ that undergoes constant remodeling and a balance between osteoblastic and osteoclastic activities is required to maintain bone homeostasis. The primary clinical sign of radiation damage to bone is local tissue atrophy, characterized by loss of functional osteoblasts, marrow adiposity, and microvascular impairments $[4,6]$. Preclinical and cell culture studies indicate that radiation damages bone formation by decreasing osteoblast number, arresting their cell cycle progression, altering their differentiation ability, and sensitizing them toward apoptosis signals [7-10]. By contrast, the radiation effect on osteoclasts is still under debate and animal studies have yielded conflicting results. While some studies clearly showed that radiation at a high dose diminishes osteoclast number within a week [11-13], other reports indicated an increase in osteoclast number as early as 3 days after whole-body irradiation [14-16] and found that anti-resorptive agents, such as risedronate and zoledronic acid, prevent radiation-induced bone loss in mice $[17,18]$. In addition, one study observed a drastic decrease in osteoclast number followed by a quick rebound in irradiated bone area in a rat model [19].

Intermittent injection of recombinant 1-34 amino-terminal fragment of parathyroid hormone (PTH1-34) is the only FDA-approved treatment for osteoporosis that stimulates both bone formation and resorption with a greater effect on bone formation. One of its anabolic mechanisms is through its suppressive action on the apoptosis of mature osteoblasts. 
Previous studies showed that PTH treatment attenuates the apoptosis of mature osteoblasts lining the trabecular bone surface in rodents under normal $[20,21]$ and pathological conditions, such as diabetes and steroid hormone treatment [22, 23]. Interestingly, Koh et al. have reported that whole-body radiation at a low dosage augments the anabolic effect of PTH on bone in mouse pups [24].

We previously reported that daily injections of PTH1-34 prevent the adverse effects of radiation resulted from multiple longitudinal microcomputed tomography $(\mu \mathrm{CT})$ scans on the trabecular bone architecture in young rats [25]. However, this study had limitations. First, $\mu \mathrm{CT}$ scan does not mimic clinical irradiator because of its low and fixed dosage. Second, the 1-month-old rats used in this study are adolescent and do not represent most adult patients receiving radiotherapy. In the present study, we investigated the therapeutic effect of PTH1-34 on a clinically relevant radiation model by using adult rats and a newly available small animal radiation research platform (SARRP) that replicates focal clinical radiotherapy in small animals [26]. A unique 3D image registration approach that we recently developed [27] was applied to accurately trace the same trabecular bone before and after radiation and PTH treatments to provide information of structural changes. Combined with mechanistic cellular changes assessed by histomorphometry and histology, our studies offer proof-of-principle evidence that PTH1-34 can minimize or fully rescue bone loss associated with radiotherapy.

\section{Materials and Methods}

\section{Rat study design}

All animal studies performed in this report were reviewed and approved by the Institutional Animal Care and Use Committee (IACUC) at the University of Pennsylvania. Three-monthold female Sprague Dawley rats (Charles River, Wilmington, MA) received a total of 16 Gy radiation fractionated as two 8 Gy doses delivered on days 1 and 3 to the proximal metaphyseal region of the right tibiae using SARRP (Xstrahl, Suwanee, GA). The radiation was delivered in a 13-mm circular collimated field centered at the metaphysis about $4 \mathrm{~mm}$ below the growth plate at a rate of $1.65 \mathrm{~Gy} / \mathrm{min}$ with the aid of build-in $\mu \mathrm{CT}$ and $\mathrm{X}$-ray. Rats were then divided into two groups ( $\mathrm{n}=5 /$ group) with similar body weight at the outset of the study, receiving either vehicle (saline) or human recombinant PTH(1-34, $60 \mu \mathrm{g} / \mathrm{kg} / \mathrm{day}$, Bachem, Bubendorf, Switzerland) daily subcutaneous injections from day 1 for 28 days. In vivo $\mu \mathrm{CT}$ of both tibiae were performed at baseline (day 0 ) and on day 28 prior to euthanasia. Tibiae were then harvested for histomorphometric analysis. Rats were injected subcutaneously with $15 \mathrm{mg} / \mathrm{kg}$ calcein (Sigma Aldrich, St. Louis, MO) at 2 and 9 days before necropsy for dynamic bone analysis. The detailed experimental scheme is depicted in Figure 1. Tibiae from another set of irradiated rats ( $n=3 /$ time point/group) were harvested at day 14 and 28 for histology. To test the role of bone resorption, a third set of irradiated rats were divided into four groups ( $\mathrm{n}=5 /$ group) receiving 1 ) vehicle (saline, daily); 2 ) alendronate (100 $\mu \mathrm{g} / \mathrm{kg}$, twice a week); 3) PTH1-34 (60 $\mu \mathrm{g} / \mathrm{kg} / \mathrm{day}) ; 4)$ PTH1-34 (60 $\mu \mathrm{g} / \mathrm{kg} / \mathrm{day})$ and alendronate $(100 \mu \mathrm{g} / \mathrm{kg}$, twice a week). Bilateral tibiae were harvested at day 28 for $\mu \mathrm{CT}$ analysis. Serum was collected after euthanasia to assess TRACP $5 \mathrm{~b}$ and osteocalcin levels 
by RatTRAPTM Assay (Immunodiagnostic Systems, Scottsdale, AZ) and Rat osteocalcin ELISA kit (Biomedical Technologies, Ward Hill MA), respectively.

\section{In vivo $\mu C T$ scan and analysis}

In vivo $\mu \mathrm{CT}$ scan, image analysis of trabecular bone, and finite element analysis (FEA) were performed as previously described [27]. Briefly, rats were anesthetized by isoflurane and their right tibiae were immobilized with a customized holder for scanning by an in vivo $\mu \mathrm{CT}$ system (vivaCT 40, Scanco Medical AG, Brüttisellen, Switzerland) at $10.5 \mu \mathrm{m}$ nominal voxel size. Bone voxels of each image were segmented from bone marrow and background using Gaussian filtering ( sigma $=1.2$ and support $=2.0$ ) and a global threshold corresponding to $495 \mathrm{mmHA} / \mathrm{cm}^{3}$. An adaptive thresholding technique was used to determine a suitable segmentation threshold for all rats in this study. For 3D image registration, a landmark-initialized, mutual-information-based registration toolkit [28, 29] of an open source software (National Library of Medicine Insight Segmentation and Registration Toolkit, USA) [30] was used to register the scans of the proximal tibia at day 0 and day 28. According to the registration, the longitudinal growth rate was determined for each bone. Trabecular bone parameters and stiffness were calculated from the area 2.5-4 $\mathrm{mm}$ below the growth plate at day 0 and its corresponding area at day 28 .

\section{Static and dynamic histomorphometry}

Left and right tibiae from SARRP-treated rats were dissected at the time of necropsy and processed for methyl methacrylate embedding. Using a Polycut-S motorized microtome (Reichert, Heidelberg, Germany), longitudinal sections were cut at $5 \mu \mathrm{m}$ followed by Goldner's trichrome staining for static analysis and at $8 \mu \mathrm{m}$ without staining for dynamic measurements. All images were taken with Nikon Eclipse 90i and quantified using Bioquant Osteo Software (Bioquant Image Analysis, Nashville, TN). The primary indices include the total tissue area (TV), trabecular bone perimeter (BS), trabecular bone area (BV), osteoblast surface (Ob.S), osteoblast number (Ob.N), osteoclast surface (Oc.S), single- and doublelabeled surface, interlabel width, osteocyte number, empty lacunae number, and adipocyte number (Ad.N). The percentages of osteoblast surface (Ob.S/BS), osteoblast number (Ob.N/ BS), osteoclast surface (Oc.S/BS), and mineralizing surface (MS/BS), mineral apposition rate (MAR, $\mu \mathrm{m} /$ day), and surface-referent bone formation rate (BFR/BS, $\mu \mathrm{m}^{3} / \mu \mathrm{m}^{2} /$ day) within metaphyseal region were calculated as described by Dempster et al. [31]. Growth plate thickness was calculated as an average of 12 measurements evenly distributed across the entire growth plate. Bone marrow adipocytes appear as unstained large circular regions within the marrow in Goldner's trichrome-stained sections and their number was counted manually within a $2 \mathrm{~mm}^{2}$ tissue area located at the center of tibial epiphyseal region.

\section{Histology}

Left and right tibiae were fixed in $10 \%$ neutralized buffered formalin overnight, decalcified in $10 \%$ EDTA for 21 days, and processed and embedded in paraffin. Five $\mu$ m-thick sections were either stained with hematoxylin and eosin $(\mathrm{H} \& \mathrm{E})$ for general histology or stained with Apoptag in situ peroxidase kit (EMD Millipore, Billerica, MA) for TUNEL assay to detect apoptotic osteoblasts. 


\section{Statistics}

Data are expressed as means \pm standard error (SEM) and analyzed by paired, two tail Student's t-test for comparison of the same tibiae before and after radiation and for comparison of irradiated and non-irradiated contralateral tibiae, and by unpaired Student's ttest for comparison of vehicle and PTH-treated samples followed by Bonferroni adjustment for multiple comparisons using Prism 5 software (GraphPad Software, San Diego, CA). Values of $p<0.05$ were considered statistically significant.

\section{Results}

\section{D image registration reveals local trabecular bone loss after SARRP irradiation}

To replicate clinical focal radiotherapy, we irradiated the right tibial metaphyseal region in 3-month-old rats by SARRP at a dose of 8 Gy twice (day 1 and 3). For this dose fractionation schedule, the biologically equivalent dose in $2 \mathrm{~Gy}$ fractions assuming an $\alpha / \beta$ of 3 for late toxicity is approximately $35 \mathrm{~Gy}$. This was designed to mimic the typical femur dose constraints for whole pelvis intensity modulated radiotherapy for patients with prostate, rectal, or endometrial cancers. The contralateral tibiae that did not receive radiation, served as paired controls since their trabecular parameters are similar to age- and gender-matched left tibiae in normal rats never receiving any radiation exposure (Supplemental Table). Rats were then separated into two groups, receiving either vehicle or PTH1-34 injections for 4 weeks. At the end of treatment, there were no significant difference in body weight among vehicle-(259.6 $\pm 5.9 \mathrm{~g})$, PTH- $(259.3 \pm 4.3 \mathrm{~g})$ treated rats and their age- and gender-matched radiation naive rats $(266.5 \pm 5.0)$, implicating that SARRP focal radiation does not have obvious systemic effects.

Unlike large mammals, rats do not close their growth plates or cease their longitudinal bone growth at skeletal maturity. Since the tibial growth plate was within the radiation field, histological staining shows that SARRP radiation enlarged the growth plate and caused disorganization of its columnar chondrocyte arrangement (Fig. 2A, B). These changes led to a significant 57\% reduction in the longitudinal growth rate in irradiated right tibia compared to non-irradiated left tibia (Fig. 2C). PTH treatment had no effect on this growth damage caused by SARRP radiation (Fig. 2). The standard $\mu \mathrm{CT}$ approach measuring the trabecular bone parameters analyzes a metaphyseal area at $2.5-4 \mathrm{~mm}$ below the tibial growth plate. However, due to their different longitudinal growth rate, the same metaphyseal areas in nonirradiated and irradiated tibiae at day 0 would move to different regions in terms of their distance to the growth plate at day 28. To overcome this growth issue that does not exist in adult patients receiving radiotherapy, we performed in vivo $\mu \mathrm{CT}$ scans on bilateral tibiae twice, at day 0 (before radiation) and day 28 (after radiation), and applied a unique 3D image registration method recently developed by our group to identify the trabecular structure at day 28 that matches the metaphyseal area at $2.5-4 \mathrm{~mm}$ below the growth plate at day 0 in the same tibia (Fig. 3A). This approach made it possible to accurately track the local changes in the same block of trabecular bone after radiation.

Careful examination of the reconstructed 3D $\mu \mathrm{CT}$ images revealed that, while almost all trabecular elements were preserved in non-irradiated samples during 4 weeks of growth, 
some small trabecular elements were apparently lost after radiation (red circles in Fig. 3B), implying that radiation shifts the balance of bone remodeling toward more resorption.

Further calculation of structural parameters confirms this observation. As shown in Fig. 3C, trabecular bone mineral density (BMD) and bone volume fraction (BV/TV) in non-irradiated tibiae of vehicle-treated rats at day 28 increased dramatically ( $21 \%$ and $51 \%$, respectively) with increased trabecular thickness (Tb.Th, 30\%), unchanged trabecular number (Tb.N) and trabecular separation ( $\mathrm{Tb} . \mathrm{Sp}$ ) compared to its own at day 0. By contrast, trabecular BMD and BV/TV in irradiated tibiae of vehicle-treated rats at day 28 only increased modestly (10\% and $29 \%$, respectively) compared to its own at day 0. Compared to non-irradiated contralateral tibiae, BMD and BV/TV from irradiated tibiae were $9 \%$ and $14 \%$ less, respectively, at day 28 , which was accompanied by a $14 \%$ decrease in Tb.N. The increased structure model index (SMI) in irradiated trabecular bone compared to non-irradiated bone in vehicle-treated rats at day 28 further confirms the radiation damage to the microarchitecture of trabecular bone. In addition, analyzing the mechanical property of trabecular bone by FEA revealed a 51\% lower trabecular bone stiffness in irradiated bones compared to non-irradiated controls (Fig. 3D), suggesting that loss of small trabecular elements leads to severely impaired mechanical strength after radiation.

\section{PTH1-34 alleviates the local loss of trabecular elements induced by SARRP radiation}

Interestingly, a close look at the 3D registered $\mu \mathrm{CT}$ images of PTH-treated bones uncovered that radiation-induced loss of small trabecular elements did not occur in these rats at day 28 (Fig. 3B). PTH treatment had a great anabolic effect on trabecular bone and increased bone mass in both non-irradiated and irradiated tibiae substantially to a similar level (Fig. 3C). This was achieved by increasing Tb.Th in both non-irradiated and irradiated tibiae and maintaining Tb.N in irradiated tibiae, together with decreasing SMI regardless of radiation. FEA further indicated that trabecular bone strength was maintained in irradiated bones after PTH treatment (Fig. 3D). Taken together, these data demonstrate the remarkable ability of PTH1-34 to alleviate radiotherapy-induced local trabecular bone loss and strength deterioration.

\section{The preservation of osteoblasts and osteocytes is important for the rescue effect of PTH1- 34 on SARRP radiation-induced bone loss}

To understand the cellular mechanisms by which PTH1-34 rescues bone damage after SARRP radiation, histological analysis was performed on irradiated and contralateral tibiae. As shown in Fig. 4A, SARRP radiation greatly damaged bone marrow cells, leading to a decrease in marrow cellularity and an increase in vessel swelling. PTH treatment did not appear to reverse this damage. Interestingly, while the majority of trabecular bone surfaces in non-irradiated samples were covered with either bone lining cells or osteoblasts, few bone surface cells, particularly osteoblasts, were observed in irradiated bone after vehicle treatment (Fig. 4A, B). By contrast, PTH-treated irradiated bones had more than a 3.9-fold increase in functional osteoblast number (Ob.N/BS) compared to vehicle-treated irradiated bones (Fig. 4A, B). Dynamic histomorphometry measurement demonstrated a drastic $78 \%$ decrease in mineral surface (MS/BS) and a nearly complete absence of calcein doublelabeled surface in irradiated bones with vehicle treatment (Fig. 4C). However, PTH treatment was able to recover the MS/BS, mineral apposition rate (MAR), and bone 
formation rate (BFR) to a similar level as non-irradiated bone with vehicle treatment. These data clearly indicate that PTH treatment protects the number and activity of osteoblasts from radiation damage.

We also found that radiation reduced osteoclast number, which was not rescued by PTH treatment (Fig. 4B). To further investigate the role of bone resorption in these events, we treated rats with alendronate, an osteoclast inhibitor, right after SARRP radiation for 4 weeks. Biochemical analysis of serum bone resorption (TRAP) and formation (osteocalcin) markers confirmed that alendronate suppressed osteoclast activity regardless of PTH treatment and that PTH1-34 elevated osteoblast activity regardless of alendronate treatment (Fig. 5A). Interestingly, $\mu \mathrm{CT}$ analysis revealed that alendronate did not block either radiation-induced trabecular bone loss or the rescue effect of PTH (Fig. 5B). These data strongly suggest that bone formation rather than bone resorption is crucial in mediating radiation damage to bone structure and support the idea that bone anabolic agent should be considered when treating radiotherapy-induced osteoporosis.

\section{PTH1-34 reduces radiation-induced apoptosis in osteoblasts and osteocytes}

Loss of functional osteoblast is one of the characteristics of radiation damage to bone. To test whether radiation causes osteoblast apoptosis in vivo and whether PTH1-34 protects osteoblasts from such cell death, we harvested tibiae at 2 weeks after radiation for TUNEL staining. As shown in Fig. 6A and B, normal bone had a low level of apoptosis (5\%) in osteoblasts lining trabecular bone surfaces but radiation resulted in a remarkable 6.9-fold increase to $34 \%$ in the vehicle-treated rats. PTH treatment greatly attenuated such damaging effects on osteoblasts and reduced the apoptosis rate to $10 \%$. Although osteocytes are relatively radio-resistant compared to osteoblasts, we noticed similar trends in osteocyte apoptosis under radiation and PTH treatment condition (data not shown). At 4 weeks after radiation, we observed that radiation resulted in a significant 4.4-fold increase in the percentage of empty lacunae, a sign of osteocyte cell death, in the vehicle-treated bone (Fig. 6C, D). Similarly, PTH treatment largely blocked such increase, indicating that PTH is able to protect not only osteoblasts but also osteocytes from radiation-induced cell death.

\section{PTH1-34 partially alleviates radiation-induced bone marrow adiposity}

One hallmark for bone marrow damage caused by radiotherapy is increased marrow adiposity. In our focal radiation model, we found a remarkable increase in adipocyte number in bone marrow within the irradiated area (Fig. 7A, B). Quantification of adipocyte number was performed in the tibial epiphyseal region because adipocytes can be morphologically identified as unstained circular regions in the bone marrow. Interestingly, while radiation led to a 3.8-fold increase in the number of bone marrow adipocytes per tissue area (Ad.N/TV), PTH1-34 partially but significantly alleviated this increase, resulting in a $41 \%$ decrease in bone marrow adiposity. These results prove that PTH1-34 inhibits radiation-induced adipogenesis of bone marrow mesenchymal progenitors, which could be another mechanism through which PTH maintains bone structure and osteoblasts after radiation. 


\section{Discussion}

In this study, we established a radiation-induced bone damage model by focally radiating rat tibiae with high accuracy at a therapeutic dose using a newly available irradiator (SARRP) that replicates the focal radiation therapy received by cancer patients. This model mimics many aspects of radiotherapy damage on bone, such as loss of mineralized bone, decreased marrow cellularity, loss of vascular integrity, and marrow adiposity. Using this clinically relevant model, we studied radiation-induced trabecular bone loss and its rescue by PTH134 injections. Structural analyses tracing the same trabecular bone before and after radiation demonstrated that radiation induces the loss of small trabeculae, leading to significant reductions in bone mass and mechanical strength, and that PTH1-34 injections are able to reverse this detrimental effect on bone microarchitecture. Histomorphometry and histology further proved that radiation drastically damages osteoblast and this damage can be partially reversed by PTH treatment. We also observed a decrease in osteoclast number after radiation. The imbalance of radiation effects on these bone cells might lead to relatively higher osteoclast activity compared to osteoblast activity after radiation, which could have then caused a loss of small but not large trabecular elements as revealed by 3D image registration. Osteoblasts are short-lived cells that need to be constantly replenished from mesenchymal progenitors. Indeed, radiation also shrinks the population of bone marrow mesenchymal progenitors [13, 25], resulting in bone damage in patients for many years. By protecting osteoblasts and osteocytes from radiation-induced cell death, PTH1-34 could have the ability to restore a favorable bone marrow microenvironment for these progenitors and therefore have a long-term beneficial effect.

A unique strength of the current study is the longitudinal evaluation of the local changes on the same trabecular structure over the treatment period. In rodents, the changes in bone structure due to altered local bone activities are often confounded with those resulting from continuous linear growth of long bones. Since radiation reduces longitudinal bone growth, we used a specialized image registration and processing scheme, in conjunction with a customized in vivo $\mu \mathrm{CT}$ imaging protocol, to maximize the accuracy of detecting local bone structural changes. This technique allows us to exclude the confounding growth issues in rodents so that the outcomes can be applicable to human. By utilizing this novel technology with a high image resolution, we were able to track individual trabecular elements over time to demonstrate the structural mechanisms of radiation damage and PTH's anabolic effect. Many previous reports [25, 32-34], including ours, have shown that PTH increases trabecular number by using cross-sectional comparison to interrogate changes in the trabecular structure. However, by longitudinal examination of registered trabecular bone images over time, we identified the thickening of existing trabeculae as the major microstructural mechanism of PTH's anabolic action. This technique also allowed us to determine that, in contrast to the loss of trabecular elements in vehicle-treated bones, main trabecular elements and their connections are well preserved in PTH-treated ones after radiation.

Previous rodent studies demonstrated that radiation resulted in a marked decrease in trabecular bone mass starting from 2 weeks and persisting over $2-3$ months $[14,15,18,35-$ 38]. Histomorphometry suggested that decreased osteoblast activity [13, 18, 35, 37] and 
increased osteoclast activity [14-16] were possible causes of this bone loss. Accordingly, risedronate, an anti-resorptive agent, was found to be effective in blocking this bone loss [18]. However, these studies exposed either the entire or a large portion of the body to radiation, which is fundamentally different from the focal radiation used in the clinic due to two reasons. First, the radiation dose used in these studies was much lower ( 2 Gy) than focal radiotherapy (20-80 Gy) due to a low tolerance of rodents toward whole body radiation (4-10 Gy). Second, as indicated by reduced body weight and organ size [24, 35, 37], total-body radiation causes systemic side effects, which could have indirect consequences on osteoclast. A recent report [17] circumvented most of these problems by radiating entire right limb of mice at $20 \mathrm{~Gy}$ and found that zoledronic acid, another antiresorptive agent, blocks trabecular bone loss after radiation. Yet no histomorphometry data were presented in this study. In line with other reports [6, 11-13], we found that focal radiation at a high dose diminishes both osteoblasts and osteoclasts at 4 weeks postradiation. Although some of those studies have shown that radiation decreases osteoclast number as early as 3-7 days [11-13], we cannot completely exclude the possibility that in our experiment an early rise of osteoclast number may have contributed to radiation-induced bone loss. Nevertheless, we found that co-treatment of alendronate does not attenuate SARRP radiation-induced bone loss or abolish the rescue effect of PTH1-34. We also demonstrated that the rescue effect of PTH1-34 on radiation-induced bone damage is dependent on its ability to protect osteoblasts but not osteoclasts after radiation. These data strongly argue that diminished bone formation but not enhanced bone resorption is a major contributor to post-radiation bone loss.

The present study provides proof-of-principle evidence that anabolic treatment that preserves osteoblast lineage cells and stimulates bone formation is a possible therapy for radiotherapy-induced osteoporosis. However, due to the increased incidence of osteosarcoma in preclinical rodent studies [39,40], there are considerable concerns in using PTH in cancer patients that have received radiation therapy. Nevertheless, results from a decade of clinical treatment of osteoporosis by PTH failed to establish a causal relationship between PTH and adult osteosarcoma [41, 42]. Moreover, long-term (3-4 years) clinical trials of PTH1-34 and 1-84 in children and adults with hypo-parathyroidism have not been associated with osteosarcoma or other bone tumors [43, 44]. These clinical observations significantly minimize the concerns of using PTH in cancer patients and promote more exploratory studies in this field. Indeed, PTH is currently investigated as a therapeutic treatment for mandible bone regeneration in head and neck cancer patients after surgery and radiotherapy because it reverses radiation-induced damage and enhances bone regeneration in a murine mandibular model of distraction osteogenesis [45]. Further studies to understand the molecular mechanisms of the pro-survival effect of PTH on osteoblasts and osteocytes will not only provide insights for the therapeutic PTH treatment but also help to identify additional therapies with fewer concerns.

\section{Supplementary Material}

Refer to Web version on PubMed Central for supplementary material. 


\section{Acknowledgments}

This work was supported by the National Institute of Health (R01DK095803 to LQ), Penn Center for Musculoskeletal Disorders P30AR050950 (NIAMS/NIH), ASBMR Junior Faculty Osteoporosis Basic Research Award (to LQ) and McCabe Pilot Award (to XSL).

\section{Reference}

1. Ries, LAG.; Eisner, MP.; Kosary, CL.; Hanke, BF.; Miller, BA.; Clegg, L.; Mariott, A.; Feuer, EJ.; Edwards, BK. SEER Cancer Statistics Review, 1973-1999. Bethesda, MD: National Cancer Institute; 2002.

2. Perez, CA.; Halperin, EC.; Brad, LW.; Wazer, DE. Perez \& Brady's Principles and Practice of Radiation. 6th ed. Philadelphia, PA, USA: Wolters Kluwer Lippincott Williams and Wilkins; 2013.

3. Costantino PD, Friedman CD, Steinberg MJ. Irradiated bone and its management. Otolaryngol Clin North Am. 1995; 28:1021-1038. [PubMed: 8559570]

4. Howland WJ, Loeffler RK, Starchman DE, Johnson RG. Postirradiation atrophic changes of bone and related complications. Radiology. 1975; 117:677-685. [PubMed: 1188119]

5. Williams HJ, Davies AM. The effect of X-rays on bone: a pictorial review. Eur Radiol. 2006; 16:619-633. [PubMed: 16237551]

6. Ergun H, Howland WJ. Postradiation atrophy of mature bone. CRC Crit Rev Diagn Imaging. 1980; 12:225-243. [PubMed: 6985580]

7. Dudziak ME, Saadeh PB, Mehrara BJ, Steinbrech DS, Greenwald JA, Gittes GK, Longaker MT. The effects of ionizing radiation on osteoblast-like cells in vitro. Plast Reconstr Surg. 2000; 106:1049-1061. [PubMed: 11039376]

8. Gal TJ, Munoz-Antonia T, Muro-Cacho CA, Klotch DW. Radiation effects on osteoblasts in vitro: a potential role in osteoradionecrosis. Arch Otolaryngol Head Neck Surg. 2000; 126:1124-1128. [PubMed: 10979127]

9. Matsumura S, Jikko A, Hiranuma H, Deguchi A, Fuchihata H. Effect of X-ray irradiation on proliferation and differentiation of osteoblast. Calcif Tissue Int. 1996; 59:307-308. [PubMed: 8781058]

10. Szymczyk KH, Shapiro IM, Adams CS. Ionizing radiation sensitizes bone cells to apoptosis. Bone. 2004; 34:148-156. [PubMed: 14751572]

11. Gungor T, Hedlund T, Hulth A, Johnell O. The effect of irradiation on osteoclasts with or without transplantation of hematopoietic cells. Acta Orthop Scand. 1982; 53:333-337. [PubMed: 6124082]

12. Sawajiri M, Mizoe J, Tanimoto K. Changes in osteoclasts after irradiation with carbon ion particles. Radiat Environ Biophys. 2003; 42:219-223. [PubMed: 13680258]

13. Cao X, Wu X, Frassica D, Yu B, Pang L, Xian L, Wan M, Lei W, Armour M, Tryggestad E, Wong J, Wen CY, Lu WW, Frassica FJ. Irradiation induces bone injury by damaging bone marrow microenvironment for stem cells. Proc Natl Acad Sci U S A. 2011; 108:1609-1614. [PubMed: 21220327]

14. Kondo H, Searby ND, Mojarrab R, Phillips J, Alwood J, Yumoto K, Almeida EA, Limoli CL, Globus RK. Total-body irradiation of postpubertal mice with (137)Cs acutely compromises the microarchitecture of cancellous bone and increases osteoclasts. Radiat Res. 2009; 171:283-289. [PubMed: 19267555]

15. Kondo H, Yumoto K, Alwood JS, Mojarrab R, Wang A, Almeida EA, Searby ND, Limoli CL, Globus RK. Oxidative stress and gamma radiation-induced cancellous bone loss with musculoskeletal disuse. J Appl Physiol. 2009; 108:152-161. [PubMed: 19875718]

16. Willey JS, Lloyd SA, Robbins ME, Bourland JD, Smith-Sielicki H, Bowman LC, Norrdin RW, Bateman TA. Early increase in osteoclast number in mice after whole-body irradiation with 2 Gy X rays. Radiat Res. 2008; 170:388-392. [PubMed: 18763868]

17. Keenawinna L, Oest ME, Mann KA, Spadaro J, Damron TA. Zoledronic acid prevents loss of trabecular bone after focal irradiation in mice. Radiat Res. 2013; 180:89-99. [PubMed: 23772924] 
18. Willey JS, Livingston EW, Robbins ME, Bourland JD, Tirado-Lee L, Smith-Sielicki H, Bateman TA. Risedronate prevents early radiation-induced osteoporosis in mice at multiple skeletal locations. Bone. 2010; 46:101-111. [PubMed: 19747571]

19. Margulies B, Morgan H, Allen M, Strauss J, Spadaro J, Damron T. Transiently increased bone density after irradiation and the radioprotectant drug amifostine in a rat model. Am J Clin Oncol. 2003; 26:e106-e114. [PubMed: 12902907]

20. Bellido T, Ali AA, Plotkin LI, Fu Q, Gubrij I, Roberson PK, Weinstein RS, O'Brien CA, Manolagas SC, Jilka RL. Proteasomal degradation of Runx2 shortens parathyroid hormoneinduced anti-apoptotic signaling in osteoblasts. A putative explanation for why intermittent administration is needed for bone anabolism. J Biol Chem. 2003; 278:50259-50272. [PubMed: 14523023]

21. Jilka RL, Weinstein RS, Bellido T, Roberson P, Parfitt AM, Manolagas SC. Increased bone formation by prevention of osteoblast apoptosis with parathyroid hormone. J Clin Invest. 1999; 104:439-446. [PubMed: 10449436]

22. Motyl KJ, McCauley LK, McCabe LR. Amelioration of type I diabetes-induced osteoporosis by parathyroid hormone is associated with improved osteoblast survival. J Cell Physiol. 2012; 227:1326-1334. [PubMed: 21604269]

23. Weinstein RS, Jilka RL, Almeida M, Roberson PK, Manolagas SC. Intermittent parathyroid hormone administration counteracts the adverse effects of glucocorticoids on osteoblast and osteocyte viability, bone formation, and strength in mice. Endocrinology. 2010; 151:2641-2649. [PubMed: 20410195]

24. Koh AJ, Novince CM, Li X, Wang T, Taichman RS, McCauley LK. An irradiation-altered bone marrow microenvironment impacts anabolic actions of PTH. Endocrinology. 2011; 152:45254536. [PubMed: 22045660]

25. Chandra A, Lan S, Zhu J, Zhang X, Siclari VA, Altman AR, Cengel KA, Liu XS, Qin L. PTH prevents the adverse effects of focal radiation on bone architecture in young rats. Bone. 2013; 55:449-457. [PubMed: 23466454]

26. Wong J, Armour E, Kazanzides P, Iordachita I, Tryggestad E, Deng H, Matinfar M, Kennedy C, Liu Z, Chan T, Gray O, Verhaegen F, McNutt T, Ford E, DeWeese TL. High-resolution, small animal radiation research platform with $\mathrm{x}$-ray tomographic guidance capabilities. Int $\mathrm{J}$ Radiat Oncol Biol Phys. 2008; 71:1591-1599. [PubMed: 18640502]

27. Lan S, Luo S, Huh BK, Chandra A, Altman AR, Qin L, Liu XS. 3D image registration is critical to ensure accurate detection of longitudinal changes in trabecular bone density, microstructure, and stiffness measurements in rat tibiae by in vivo microcomputed tomography (uCT). Bone. 2013; 56:83-90. [PubMed: 23727434]

28. Collignon, A.; Maes, F.; Delaere, D.; Vandermeulen, D.; Suetens, P.; Marchal, G. Automated multi-modality image registration based on information theory. In: Bizais, Y.; Barillot, C.; di Paola, R., editors. Information Processing in Medical Imaging. Dordrecht, The Netherlands: Kluwer Academic Publishers; 1995. p. 263-274.

29. Viola P, Wells WM III. Alignment by maximization of mutual information. Int J Comput Vis. 1997; 24:137-154.

30. Ibanez, L.; Schroeder, W.; Ng, L.; Cates, J. [Accessed December 5, 2008] The ITK Software Guide. 2nd Ed.Available at http://www.itk.org/ItkSoftwareGuide.pdf

31. Dempster DW, Compston JE, Drezner MK, Glorieux FH, Kanis JA, Malluche H, Meunier PJ, Ott SM, Recker RR, Parfitt AM. Standardized nomenclature, symbols, and units for bone histomorphometry: a 2012 update of the report of the ASBMR Histomorphometry Nomenclature Committee. J Bone Miner Res. 2013; 28:2-17. [PubMed: 23197339]

32. Brennan TC, Rizzoli R, Ammann P. Selective modification of bone quality by PTH, pamidronate, or raloxifene. J Bone Miner Res. 2009; 24:800-808. [PubMed: 19063681]

33. Friedl G, Turner RT, Evans GL, Dobnig H. Intermittent parathyroid hormone (PTH) treatment and age-dependent effects on rat cancellous bone and mineral metabolism. J Orthop Res. 2007; 25:1454-1464. [PubMed: 17557320]

34. Prisby R, Guignandon A, Vanden-Bossche A, Mac-Way F, Linossier MT, Thomas M, Laroche N, Malaval L, Langer M, Peter ZA, Peyrin F, Vico L, Lafage-Proust MH. Intermittent PTH(1-84) is 
osteoanabolic but not osteoangiogenic and relocates bone marrow blood vessels closer to boneforming sites. J Bone Miner Res. 2011; 26:2583-2596. [PubMed: 21713994]

35. Green DE, Adler BJ, Chan ME, Rubin CT. Devastation of adult stem cell pools by irradiation precedes collapse of trabecular bone quality and quantity. J Bone Miner Res. 2012; 27:749-759. [PubMed: 22190044]

36. Hamilton SA, Pecaut MJ, Gridley DS, Travis ND, Bandstra ER, Willey JS, Nelson GA, Bateman TA. A murine model for bone loss from therapeutic and space-relevant sources of radiation. J Appl Physiol. 2006; 101:789-793. [PubMed: 16741258]

37. Jia D, Gaddy D, Suva LJ, Corry PM. Rapid loss of bone mass and strength in mice after abdominal irradiation. Radiat Res. 2011; 176:624-635. [PubMed: 21859327]

38. Wernle JD, Damron TA, Allen MJ, Mann KA. Local irradiation alters bone morphology and increases bone fragility in a mouse model. J Biomech. 2010; 43:2738-2746. [PubMed: 20655052]

39. Vahle JL, Long GG, Sandusky G, Westmore M, Ma YL, Sato M. Bone neoplasms in F344 rats given teriparatide [rhPTH(1-34)] are dependent on duration of treatment and dose. Toxicol Pathol. 2004; 32:426-438. [PubMed: 15204966]

40. Vahle JL, Sato M, Long GG, Young JK, Francis PC, Engelhardt JA, Westmore MS, Linda Y, Nold JB. Skeletal changes in rats given daily subcutaneous injections of recombinant human parathyroid hormone (1-34) for 2 years and relevance to human safety. Toxicol Pathol. 2002; 30:312-321. [PubMed: 12051548]

41. Andrews EB, Gilsenan AW, Midkiff K, Sherrill B, Wu Y, Mann BH, Masica D. The US postmarketing surveillance study of adult osteosarcoma and teriparatide: Study design and findings from the first 7 years. J Bone Miner Res. 2012; 27:2429-2437. [PubMed: 22991313]

42. Cipriani C, Irani D, Bilezikian JP. Safety of osteoanabolic therapy: a decade of experience. J Bone Miner Res. 2012; 27:2419-2428. [PubMed: 23165426]

43. Cusano NE, Rubin MR, McMahon DJ, Zhang C, Ives R, Tulley A, Sliney J Jr, Cremers SC, Bilezikian JP. Therapy of hypoparathyroidism with PTH(1-84): a prospective four-year investigation of efficacy and safety. J Clin Endocrinol Metab. 2013; 98:137-144. [PubMed: 23162103]

44. Winer KK, Sinaii N, Reynolds J, Peterson D, Dowdy K, Cutler GB Jr. Long-term treatment of 12 children with chronic hypoparathyroidism: a randomized trial comparing synthetic human parathyroid hormone 1-34 versus calcitriol and calcium. J Clin Endocrinol Metab. 2010; 95:26802688. [PubMed: 20392870]

45. Gallagher KK, Deshpande S, Tchanque-Fossuo CN, Donneys A, Sarhaddi D, Nelson NS, Chepeha DB, Buchman SR. Role of parathyroid hormone therapy in reversing radiation-induced nonunion and normalization of radiomorphometrics in a murine mandibular model of distraction osteogenesis. Head Neck. 2013; 17:23216. 


\section{Highlights}

- Focal radiation of proximal tibia in adult rats using a clinically relevant irradiator (SARRP) causes trabecular bone loss.

- A unique 3D image registration approach demonstrates local structural and strength damage in trabecular bone after radiation.

- PTH1-34 injections reverse radiation-induced bone loss and structural deterioration by protecting osteoblasts and osteocytes from apoptosis.

- PTH1-34 treatment also attenuates radiation-induced bone marrow adiposity.

- Our data suggest anabolic treatment that preserves osteoblast lineage cells as a possible therapy for radiotherapy-induced osteoporosis. 


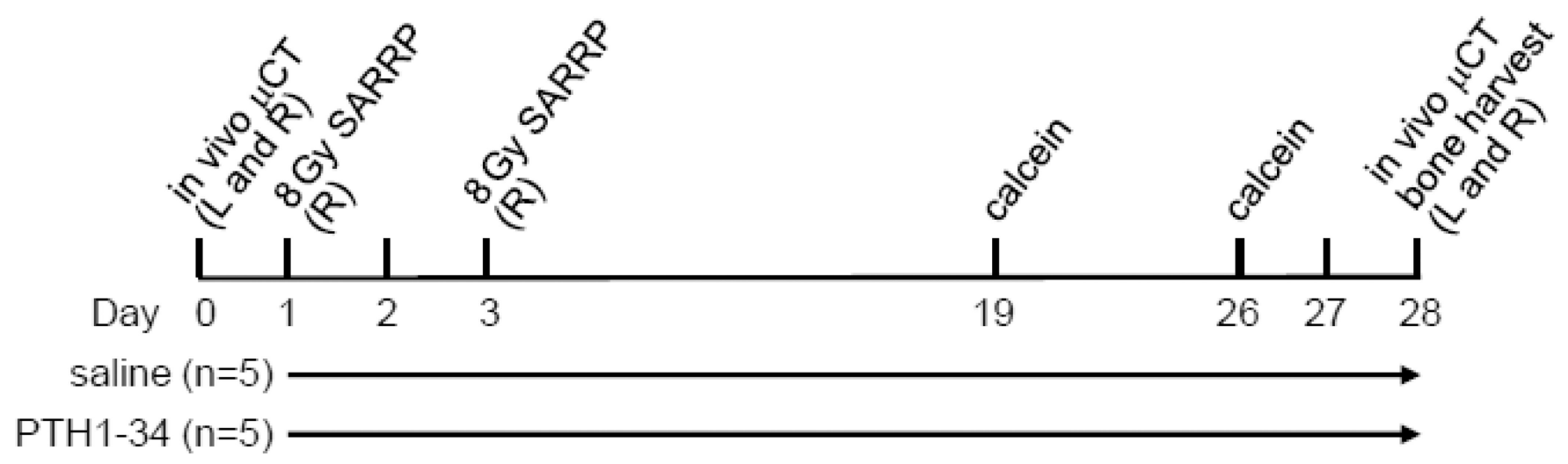

Figure 1.

A schematic representation of the experimental design to analyze the damaging effect of SARRP focal radiation on bone and the rescue effect of PTH treatment. L: left tibia; R: right tibia. 

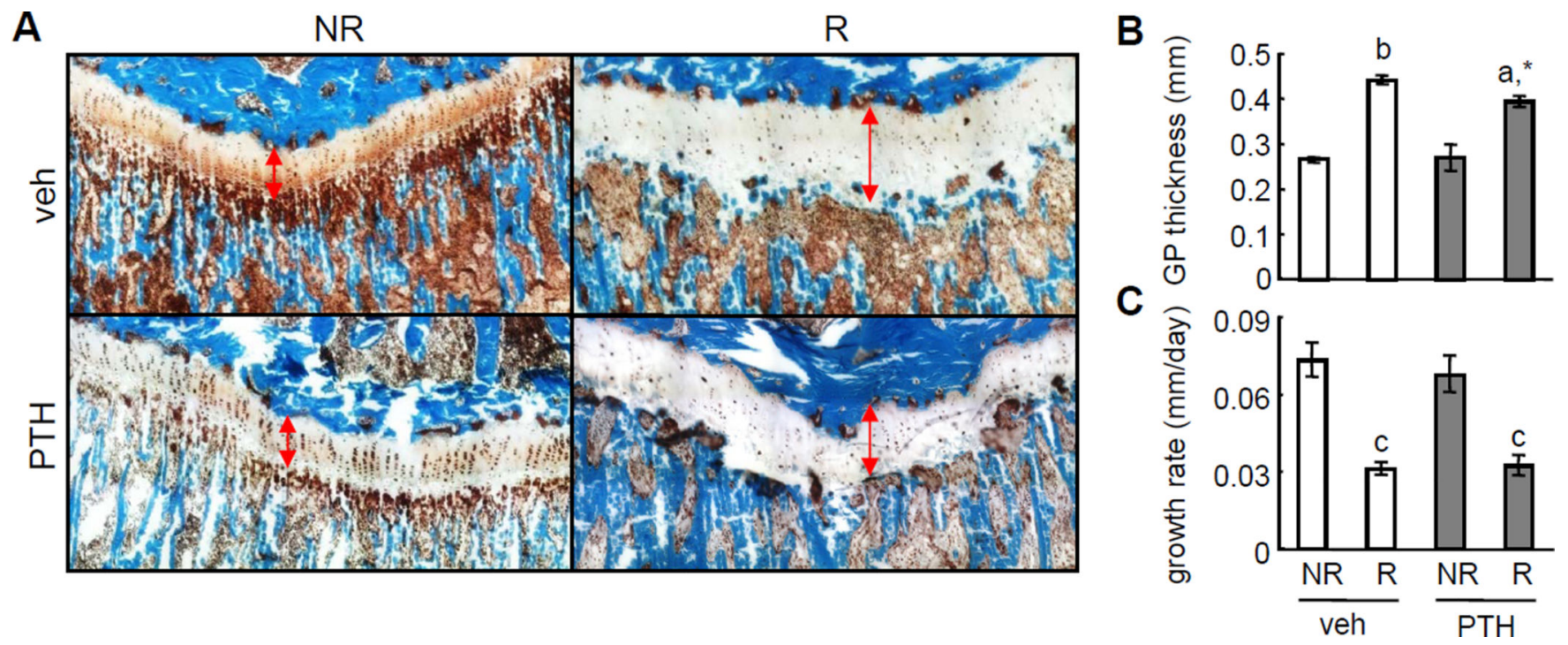

Figure 2.

Therapeutic dose of radiation impairs longitudinal bone growth regardless of PTH treatment. (A) Goldner's trichrome staining of tibial proximal growth plates at day 28 after radiation and PTH treatments. Double-arrow lines indicate the growth plate. NR: non-irradiated left tibia; R: irradiated right tibia.

(B) The average thickness of tibial growth plate was quantified.

(C) The tibial longitudinal growth rate within 28 days after radiation and PTH treatments were calculated based on $3 \mathrm{D}$ image registration.

a: $\mathrm{p}<0.05 ; \mathrm{b}: \mathrm{p}<0.01 ; \mathrm{c}: \mathrm{p}<0.001 \mathrm{R}$ vs NR. *: $\mathrm{p}<0.05$ vs veh R. 
A
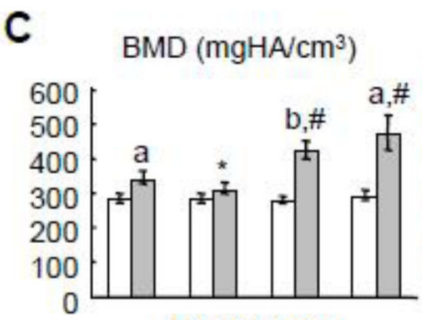

Tb. N (1/mm)

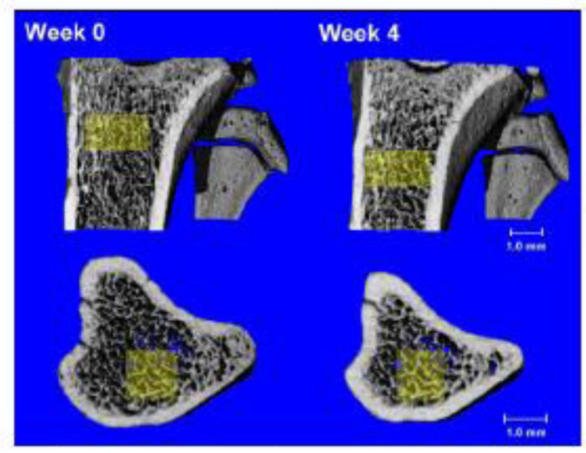

B
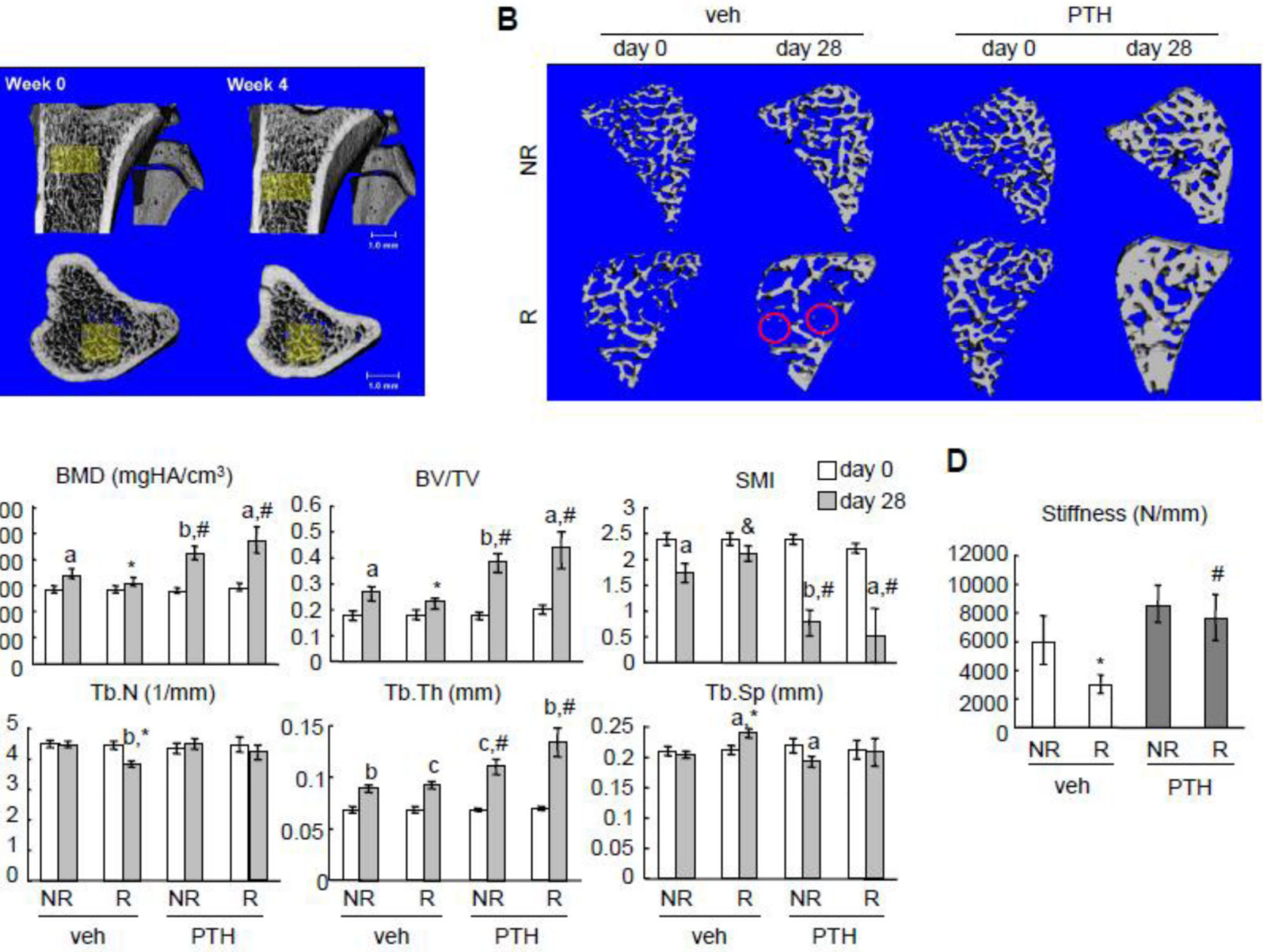

BVITV

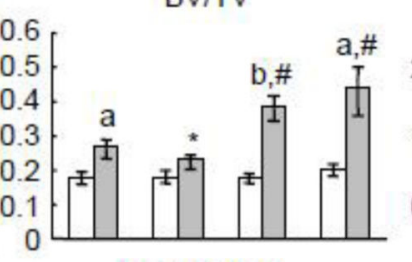

Tb.Th (mm)

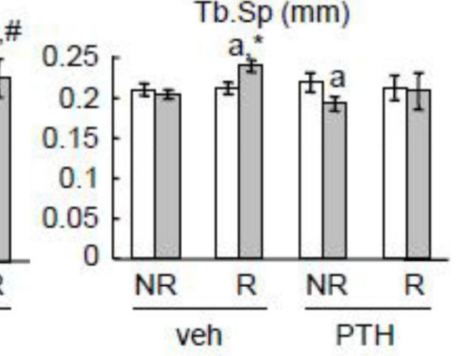

SMI $\square$ day 0

D $\square$ day 28

Figure 3.

PTH reverses trabecular bone loss and strength deterioration caused by SARRP radiation.

(A) Registered 3D whole bone images (top: anterior-posterior view; bottom: superiorinferior view) of a vehicle-treated non-irradiated rat proximal tibia at day 0 and day 28 . A common region was highlighted in yellow, showing a similar local trabecular structure in bones that were scanned 4 weeks apart.

(B) 3D reconstructed trabecular images reveals that, while some small trabecular elements (red circles) were lost after radiation, PTH treatment preserves almost all of them.

(C) $\mu \mathrm{CT}$ measurement of bone structural parameters in the same tibial trabecular area before (day 0) and after (day 28) SARRP radiation with or without PTH daily treatment. a: $\mathrm{p}<0.05$; b: $\mathrm{p}<0.01$; $\mathrm{c}: \mathrm{p}<0.001$ day 28 vs day 0; *: p<0.05 R vs NR; \#: $\mathrm{p}<0.05$ PTH vs veh.

(D) Trabecular bone stiffness was measured by $\mu \mathrm{CT}$ and FEA at day 28 . *: p<0.05 vs veh NR; \#: p<0.05 vs veh R. 


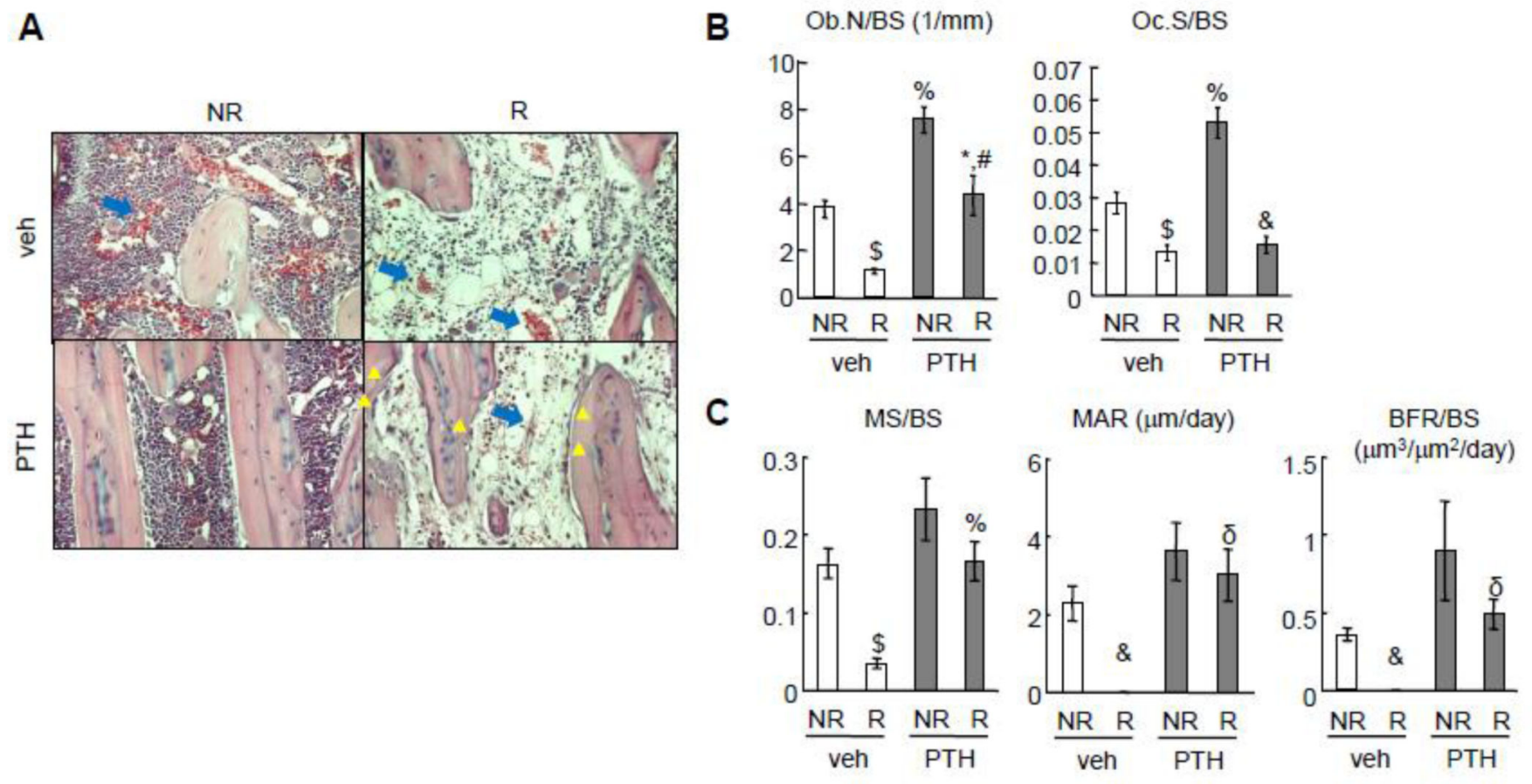

Figure 4.

PTH prevents the reductions in osteoblast number and activity caused by SARRP radiation. (A) H\&E staining of tibial trabecular bone sections at day 28 after radiation. Yellow triangles point to osteoblasts on the trabecular bone surface in the PTH group after radiation. Blue arrows point to bone marrow blood vessels.

$(B, C)$ Static (B) and dynamic (C) bone histomorphometry of tibial trabecular bone at day 28 after radiation.

*: $\mathrm{p}<0.05 ; \$: \mathrm{p}<0.01 ; \&$ : $\mathrm{p}<0.001 \mathrm{R}$ vs NR; \#: $\mathrm{p}<0.05 ; \%$ : $\mathrm{p}<0.01 ; \delta: \mathrm{p}<0.001$ PTH vs veh. 
A TRAP (unit/L)

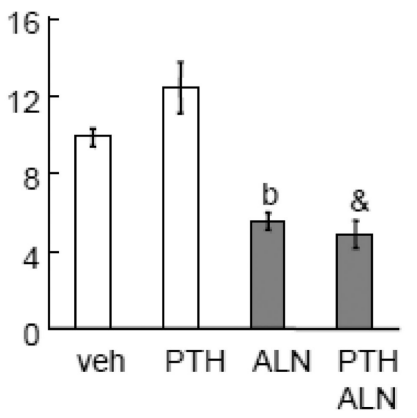

osteocalcin $(\mathrm{ng} / \mathrm{ml})$

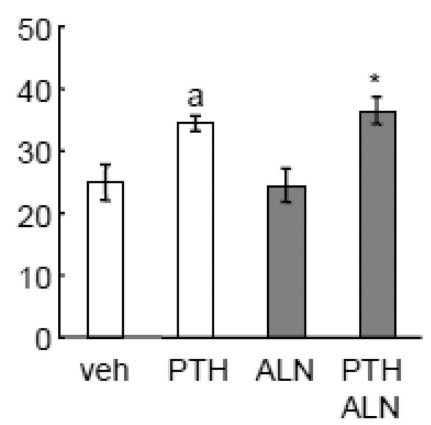

B

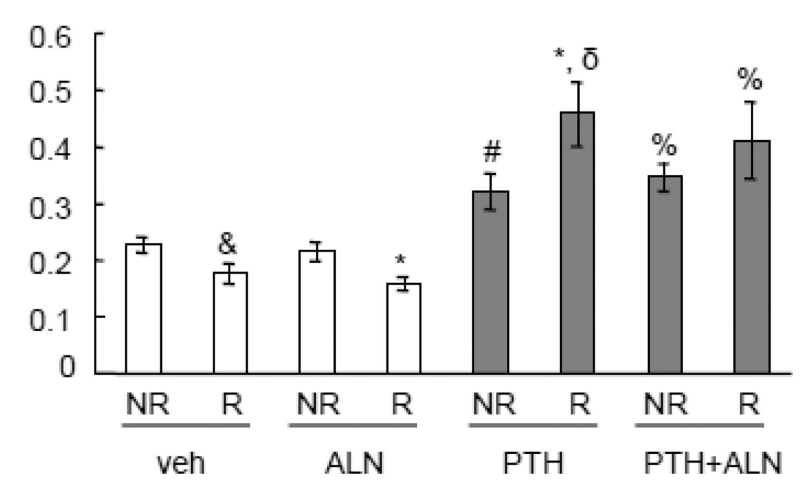

Figure 5.

Bone resorption is not essential for SARRP radiation-induced bone loss or the rescue effect of PTH.

Rats received SARRP radiation ( 8 Gy twice) to their proximal tibial metaphysis followed by vehicle, PTH, alendronate (ALN), or a combination of PTH and ALN treatment for 4 weeks.

(A) ELISA analyses of serum concentrations of bone resorption (TRAP) and formation (osteocalcin) markers at day 28 after radiation. a: $\mathrm{p}<0.05$; $\mathrm{b}$ : $\mathrm{p}<0.01$ vs veh; $\&$ : $\mathrm{p}<0.001$ vs PTH; *: p<0.05 vs ALN.

(B) $\mu \mathrm{CT}$ measurement of trabecular bone volume fraction at day 28 after radiation. *: $\mathrm{p}<0.05 ; \&$ : $\mathrm{p}<0.001$ vs its respective NR; \#: $\mathrm{p}<0.05 ; \%$ : $\mathrm{p}<0.01 ; \delta$ : $\mathrm{p}<0.001$ vs its respective veh. 
A

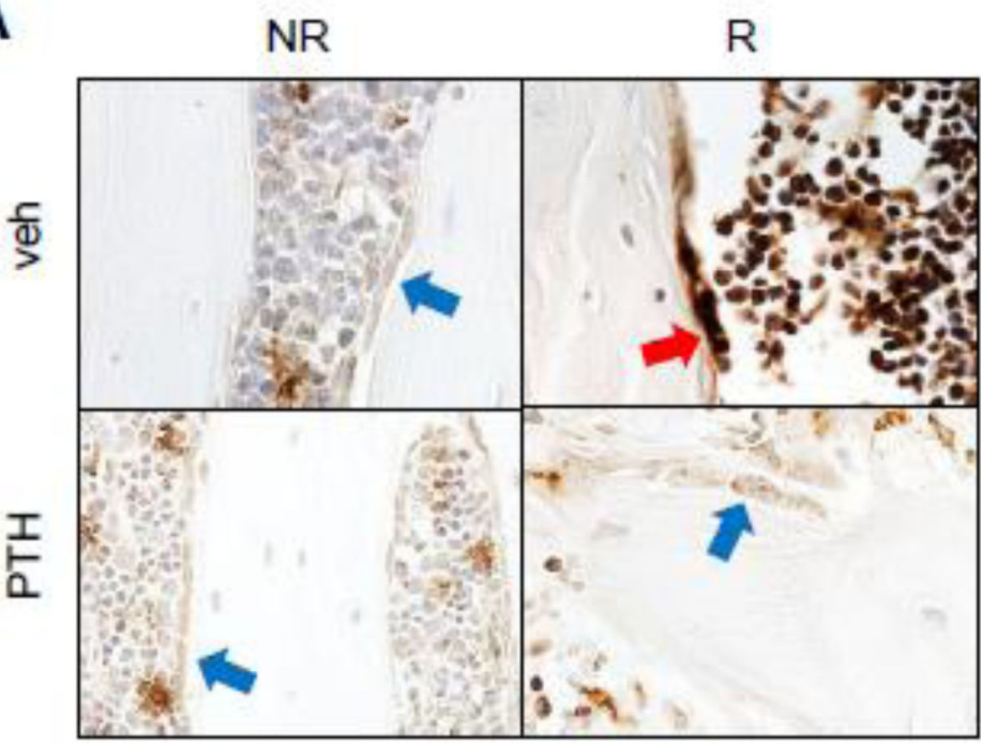

C

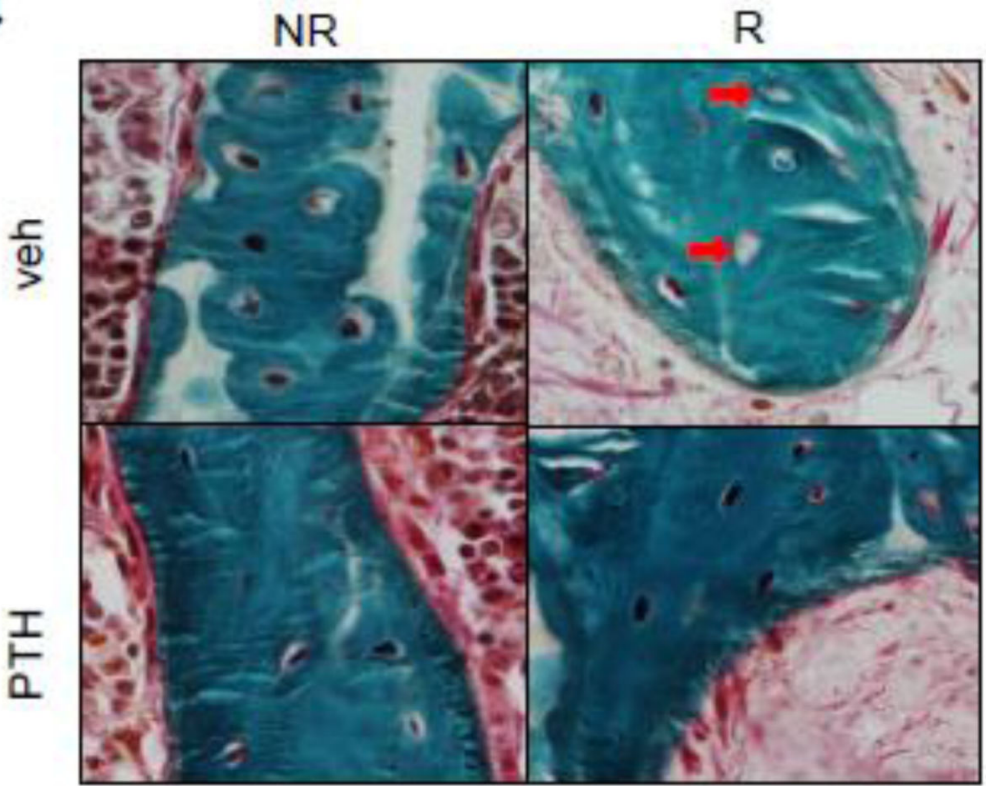

B

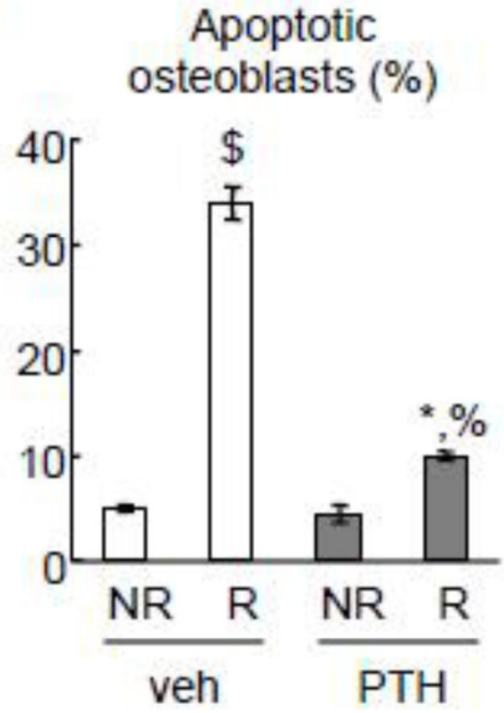

Figure 6.

PTH protects osteoblasts and osteocytes from radiation-induced cell death.

(A) TUNEL staining was performed with tibial trabecular bone sections at 2 weeks after radiation with or without PTH treatment. Red and blue arrows point to TUNEL-positive and negative osteoblasts, respectively.

(B) The percentage of apoptotic osteoblasts were quantified based on TUNEL staining.

(C) Goldner's trichrome staining of tibial trabecular bone sections at day 28 shows empty lacunae (red arrows) in the irradiated samples.

(D) The percentages of dead osteocytes were quantified. 
*: $\mathrm{p}<0.05 ;$ \$: $\mathrm{p}<0.01 \mathrm{R}$ vs NR; \%: $\mathrm{p}<0.01$ PTH vs veh. 

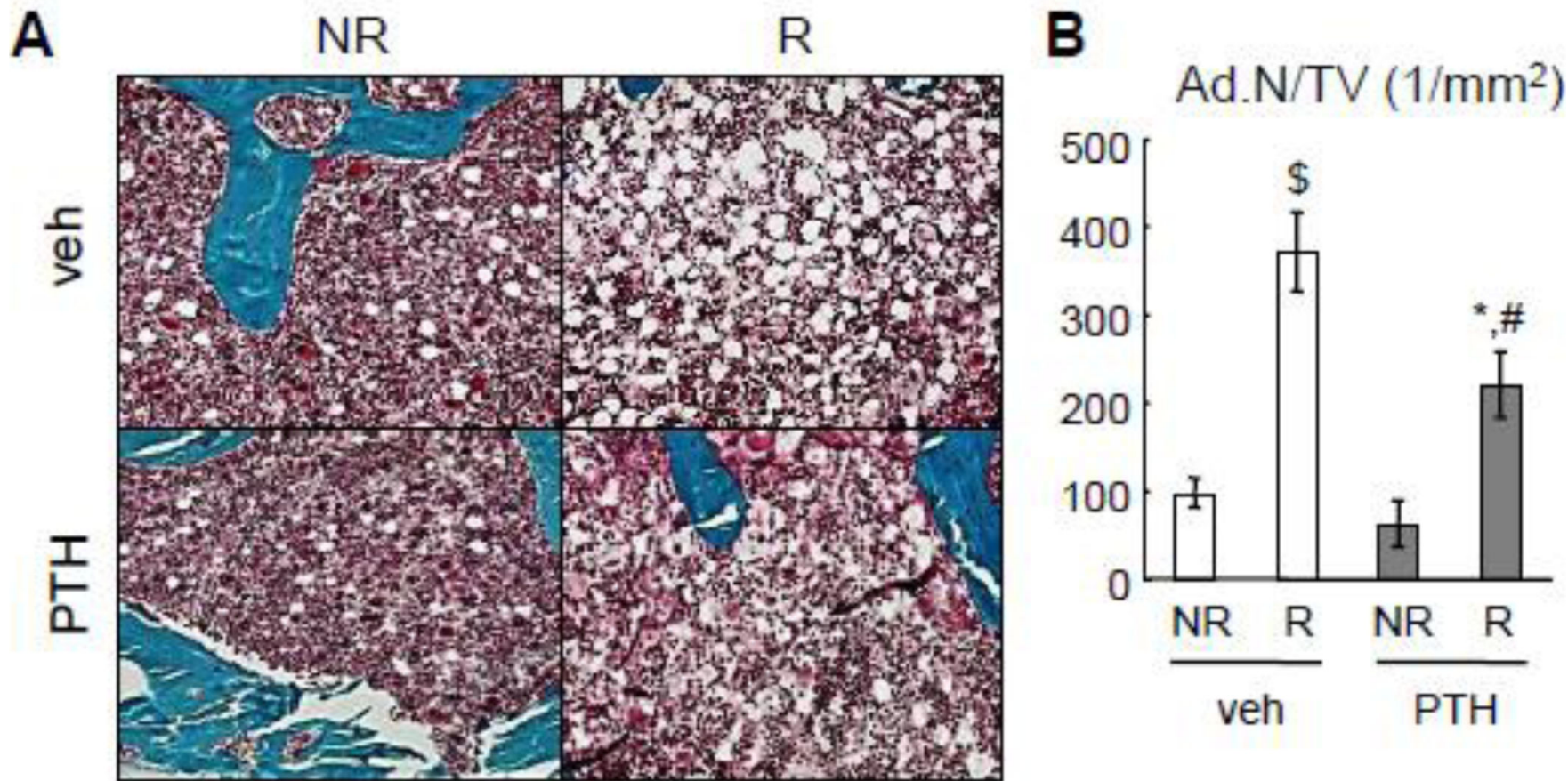

Figure 7.

PTH attenuates bone marrow adiposity caused by SARRP radiation.

(A) Goldner's trichrome staining of tibial sections at day 28 reveals bone marrow adipocytes (white circles) in the epiphyseal region.

(B) The density of bone marrow adipocytes was quantified. *: $\mathrm{p}<0.05$, \$: $\mathrm{p}<0.01 \mathrm{R}$ vs NR; \#: $\mathrm{p}<0.05$; PTH vs veh. 